\title{
Controle dinâmico de QoS baseado em políticas
}

\author{
Manoel B. C. Morais, Pedro Klécius Cardoso e Danielo G. Gomes
}

\begin{abstract}
Resumo-A convergência dos serviços e o aumento vertiginoso do tráfego na Internet nos últimos anos sugerem a busca por novos mecanismos para lidar com requisitos cada vez mais desafiadores de qualidade de serviço (QoS). Este trabalho apresenta a proposta de um controlador dinâmico, não-linear, que através do ajuste dos pesos do algoritmo WFQ em ambiente DiffServ proporciona níveis de QoS satisfatórios para as aplicações isócronas de multimídia. Os resultados alcançados pelo controlador dinâmico proposto são motivadores e, quando comparados aos do DiffServ clássico, apontam ganhos de eficiência na utilização dos recursos da rede.
\end{abstract}

Palavras-Chave - QoS, controle, WFQ, políticas.

Abstract - Converged IP-based networks and services, as well the huge increase in IP traffic along the last few years, have revealed the need for new dynamic mechanisms capable of handling all types of IP-based multimedia traffic. This work proposes a solution based on control theory running on a DiffServ domain. Other than classical solutions, whose objective is to provide strong QoS responses only for one or two premium classes, our proposal aims to provide QoS for both isochronous and best-effort applications. The results presented here show that our objective is accomplished with the correct fitting of the WFQ parameters without overestimating their weights.

Keywords - QoS control, WFQ, policies.

\section{INTRODUÇÃO}

O serviço proporcionado atualmente na Internet ainda não é adequado para atender à demanda de aplicações avançadas (e.g., videoconferência, telemedicina) e das novas aplicações que vêm surgindo com a convergência dos serviços e das mídias audiovisuais.

A arquitetura de serviços diferenciados (DiffServ) tem sido amplamente aceita na implantação de QoS em redes de comutação de pacotes IP (Internet Protocol). Essa arquitetura visa prover diferenciação de serviços de uma maneira escalável e flexível, com a classificação dos pacotes sendo realizada nos roteadores de borda da rede.

Manoel B. C. Morais e Pedro Klécius Cardoso, Centro Federal de Educação Tecnológica do Ceará (Cefet-CE), Fortaleza, Brasil; e-mails: manoelmorais@cefetce.br, klecius@cefetce.br.

Danielo G Gomes, GREat/Universidade Federal do Ceará (UFC), Fortaleza, Brasil; e-mail: dgomes@great.ufc.br.
O presente trabalho apresenta uma proposta de controle em malha fechada utilizando a arquitetura DiffServ em conjunto com o algoritmo PGPS (Packet-by-Packet Generalized Processor Sharing), também conhecido como WFQ (Weighted Fair Queueing), capaz de manter os níveis de QoS das aplicações multimídia mediante o ajuste ponderado correto dos parâmetros de peso do WFQ.

$\mathrm{O}$ diferencial da proposta consiste em manter o atraso dos fluxos controlados em um certo nível máximo, caso a rede se encontre congestionada, de modo a estabelecer um limite rígido de atraso fim-a-fim. Mantendo o atraso dos fluxos controlados em um certo nível máximo, será possível prover aos fluxos menos prioritários os recursos excedentes, melhorando assim a distribuição dos recursos da rede entre os fluxos IP, proporcionando mais justiça e eficiência no uso da rede.

$\mathrm{O}$ artigo está organizado da seguinte forma. Na Seção II são descritos os principais trabalhos relacionados à problemática aqui tratada. A Seção III apresenta sucintamente os conceitos básicos utilizados, notadamente os relativos à teoria de controle. Os cenários de estudo, bem como suas respectivas simulações preliminares à proposta, são descritos na Seção IV. Na Seção V é apresentada a proposta e os principais resultados obtidos na sua implementação são mostrados na Seção VI. Finalmente, a Seção VII traz as conclusões e algumas perspectivas futuras para esse trabalho.

\section{TRABALHOS CORRELATOS}

Várias propostas têm surgido com a finalidade de ajustar dinamicamente os parâmetros de peso do algoritmo WFQ de acordo com as condições de tráfego da rede $[5,9,10]$. Todavia, alguns algoritmos de predição do tráfego [3] ou de inteligência computacional têm apresentado limitações que dificultam sua aplicação no provimento de QoS em redes IP, seja devido ao alto custo computacional imposto aos nós da rede ou à necessidade de uma estação central de controle, o que implica em limitações de escalabilidade [4].

Dos trabalhos relacionados com o ajuste dinâmico dos pesos do algoritmo WFQ, destaca-se uma proposta de extensão ao WFQ baseada em técnicas de predição de modelo de tráfego IP [5] e a proposta de um algoritmo WFQ adaptativo denominado AWFQ, que utiliza as estimativas das taxas de chegada dos fluxos para proceder ao ajuste dos pesos [13].

Em [4], propõe-se um controlador baseado em lógica difusa (fuzzy) que reconfigura os pesos do algoritmo WFQ nos nós de um domínio Diffserv de acordo com o tráfego entrante. Este controlador foi comparado a um controlador PD (Proporcional e Derivativo) digital simples executando um controle intuitivo. Dos resultados obtidos, vale destacar 
que ao se variar o intervalo de operação dos controladores com intervalo pequeno, o controlador fuzzy é apenas um pouco melhor que o controlador convencional, tornando-se mais eficiente com intervalos maiores. O controlador fuzzy do referido trabalho apresentou um desempenho superior, mas não tão marcante em relação ao controlador convencional intuitivo.

Outro trabalho na linha da inteligência computacional, aplicada a um processo térmico de segunda ordem, compara os desempenhos de um controlador nebuloso e de um controlador PID (Proporcional-Integrador-Derivativo) otimizado através da técnica de Algoritmos Genéticos (AG) [11]. Os resultados deste trabalho mostraram que o controlador fuzzy apresentou menor tempo de estabilização, mas o controlador PID apresentou a menor variância do erro, logo os controladores PID, se bem projetados e sintonizados, constituem ainda uma boa opção de solução.

\section{CONCEITOS ASSOCIADOS}

\section{A. DiffServ e escalonamento $W F Q$}

A arquitetura de serviços diferenciados ou DiffServ, definida na recomendação 2475 do IETF, trabalha com o conceito de classes de serviço e visa agrupar fluxos de tráfego semelhantes em uma mesma classe, permitindo a redução no número de estados a serem mantidos nos roteadores de um provedor. $\mathrm{Na}$ entrada da rede, o tráfego é classificado, condicionado e integrado em uma das diferentes classes, sendo cada uma caracterizada pelo respectivo campo Differentiated Service Code Point (DSCP).

O tipo de escalonamento escolhido para ser utilizado em conjunto com a arquitetura DiffServ foi o algoritmo WFQ, pois ele permite a implementação dinâmica da divisão da largura de banda para as filas dos nós roteadores de acordo com os seus pesos; esta disciplina de enfileiramento é capaz de prover QoS e de evitar a falta de recursos aos fluxos menos prioritários (condição de inanição).

\section{B. Controladores clássicos em redes de computadores}

As políticas de gerenciamento ativo de filas ou AQM (Active Queue Management), implementadas com o auxílio da teoria de controle, utilizam na sua grande maioria os controladores clássicos do tipo PID e suas derivações $[1,7$, 14]. Por exemplo, um mecanismo de prevenção de congestionamento em redes TCP/IP, o algoritmo RED (Random Early Detection) e sua variante LRED (Loss Ratio based RED) são controladores do tipo P; o Dynamic RED (DRED) é de fato um controlador do tipo I e o PI-AQM utiliza um controlador do tipo PI.

\section{B.1. PID posicional e de velocidade}

Seja a seguinte equação do algoritmo PID posicional [2]

$u(t)=K_{c} e(t)+\frac{K_{c}}{T_{i}} T_{s} \sum_{i=0}^{t} e(i)+\frac{K_{c} T_{d}(e(t)-e(t-1))}{T_{s}}+u_{0}$,

na qual $\mathrm{u}(t)$ é a resposta do controlador, $\mathrm{K}_{\mathrm{c}}$ é o ganho crítico, $\mathrm{T}_{\mathrm{i}}$ é a constante de tempo integral, $\mathrm{T}_{\mathrm{d}}$ é a constante de tempo derivativa, $\mathrm{T}_{\mathrm{s}}$ é o intervalo de amostragem e e $(t)$ representa o erro. Aplicando-se um deslocamento regressivo no intervalo de amostragem da Equação 1, vem

$$
u(t-1)=K_{c} e(t-1)+\frac{K_{c}}{T_{i}} T_{s} \sum_{i=0}^{t-1} e(i)+\frac{K_{c} T_{d}(e(t-1)-e(t-2))}{T_{s}}+u_{0} .
$$

Subtraindo (2) de (1), obtém-se a fórmula do PID de velocidade, também conhecida como forma recursiva [2]:

$u(t)=u(t-1)+K_{c}[e(t)-e(t-1)]+\frac{K_{c} T_{s}}{T_{i}} e(t)+\frac{K_{c} T_{d}}{T_{s}}[e(t)-2 e(t-1)+e(t-2)]$

\section{B.2. Parametrização de controladores}

Ziegler e Nichols propuseram métodos heurísticos para a determinação dos valores do ganho proporcional $\mathrm{K}_{\mathrm{p}}$ bem como das constantes de tempo $\mathrm{T}_{\mathrm{i}}$ e $\mathrm{T}_{\mathrm{d}}$, baseando-se nas características da resposta transitória de uma planta [8].

As regras de Ziegler-Nichols para determinação dos parâmetros têm sido amplamente utilizadas para determinar parâmetros de controladores PID em sistemas de controle de processos nos quais a dinâmica da planta não é precisamente conhecida. As regras de Ziegler-Nichols para determinação de parâmetros podem, naturalmente, ser aplicadas a plantas cujas dinâmicas são conhecidas.

Neste trabalho, foi utilizado o segundo método de ZieglerNichols, o qual é baseado no ganho crítico em malha fechada. De acordo com este método, os valores iniciais para as constantes de tempo são $\mathrm{T}_{\mathrm{i}}=\infty \mathrm{e} \mathrm{T}_{\mathrm{d}}=0$. Usando a ação de controle proporcional, aumenta-se $\mathrm{K}_{\mathrm{p}}$ desde zero até um valor crítico $\left(\mathrm{K}_{\mathrm{c}}\right)$, no qual a saída exibe oscilações mantidas. Se a saída não exibir oscilações mantidas para quaisquer valores que $\mathrm{K}_{\mathrm{p}}$ possa assumir, então este método não se aplica. Portanto, o ganho crítico $\mathrm{K}_{\mathrm{c}}$ e o período correspondente $\mathrm{P}_{\mathrm{c}}$ são experimentalmente determinados.

Ziegler e Nichols sugerem estabelecer os valores dos parâmetros $\mathrm{K}_{\mathrm{p}}, \mathrm{T}_{\mathrm{i}}$ e $\mathrm{T}_{\mathrm{d}}$ de acordo com a fórmula mostrada na Tabela I.

TABELA I - Parâmetros Kp, Ti e Td [8].

TABELA I - Parâmetros Kp, Ti e Td [8].
\begin{tabular}{|c|c|c|c|}
\hline Controlador & $\mathrm{Kp}$ & $\mathrm{Ti}$ & $\mathrm{Td}$ \\
\hline $\mathrm{P}$ & $0,5^{\star} \mathrm{K}_{\mathrm{c}}$ & & \\
\hline $\mathrm{PD}$ & $0,65^{\star} \mathrm{K}_{\mathrm{c}}$ & & $0,12^{*} \mathrm{P}_{\mathrm{c}}$ \\
\hline $\mathrm{PI}$ & $0,45^{\star} \mathrm{K}_{\mathrm{c}}$ & $(1 / 1,2)^{*} \mathrm{P}_{\mathrm{c}}$ & \\
\hline $\mathrm{PID}$ & $0,6^{*} \mathrm{~K}_{\mathrm{c}}$ & $0,5^{*} \mathrm{P}_{\mathrm{c}}$ & $0,125^{*} \mathrm{P}_{\mathrm{c}}$ \\
\hline
\end{tabular}

\section{CENÁRIOS DE ESTUdo}

Por apresentar distribuição gratuita e ter grande aceitação na comunidade científica, o simulador escolhido para este trabalho foi o ns-2 [12].

Os cenários de simulação foram desenvolvidos com base na arquitetura de serviços diferenciados ilustrada na topologia da Figura 1. Os fluxos são gerados por servidores de fluxos de voz, vídeo, FTP e de fluxo interferente; $e l$ e $e 2$ são os roteadores de bordas da nuvem DiffServ e core representa o roteador de núcleo. As capacidades de transmissão dos enlaces entre os nós da rede são de $10 \mathrm{Mbps}$, com exceção do enlace entre o roteador core e $e 2$, cuja capacidade foi estabelecida em 1,5 Mbps para provocar um ponto de congestionamento (gargalo) na rede. $\mathrm{O}$ atraso de cada enlace é de $5 \mathrm{~ms}$. 


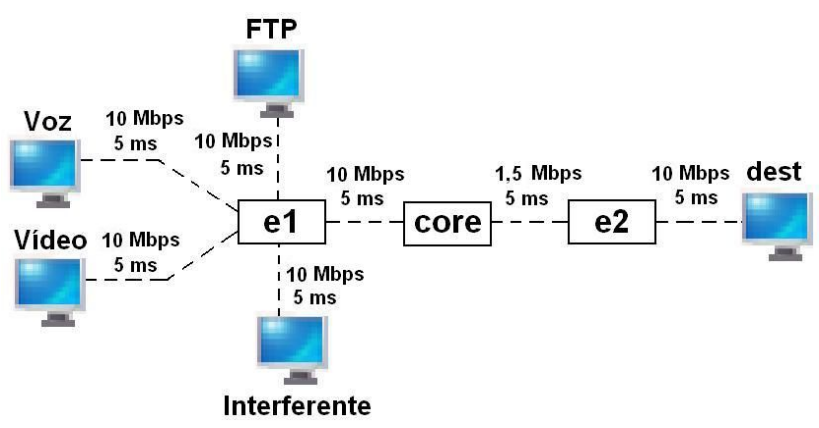

Fig. 1. Topologia de simulação.

Os fluxos de voz, de vídeo e o interferente são do tipo CBR (Constant Bit Rate), com suas taxas variando conforme as necessidades de observação da influência do congestionamento e/ou módulo de controle nos cenários desenvolvidos para análise.

\section{A. Sintonia dos controladores utilizados}

Utilizando-se o segundo método de Ziegler-Nichols para a determinação dos parâmetros dos controladores implementados, foram obtidas oscilações mantidas para o ganho de 0,08. Sendo então determinado o ganho crítico $\left(\mathrm{K}_{\mathrm{c}}=0,08\right)$ e o período das oscilações $\left(\mathrm{P}_{\mathrm{c}}=1,62\right.$ segundos $)$.

\section{B. Análise dos controladores}

Foram desenvolvidos para análise os controladores P, PI e PID a partir das Equações 1 e 3, respectivamente as equações posicional e de velocidade, sendo que cada controlador atua somente no fluxo de voz.

Os melhores resultados obtidos nas simulações preliminares, as quais deram subsídios à proposta apresentada na seção $\mathrm{V}$, foram com os controladores $\mathrm{P}$ posicional e PI velocidade. Os controladores PI posicional e PID não apresentaram resultados satisfatórios, gerando grande instabilidade e variações bruscas na variável controlada (atraso do fluxo de voz). O controlador $\mathrm{P}$ velocidade apresentou características semelhantes ao controlador $\mathrm{P}$ posicional, com desnível no valor de referência estabelecido, mas com uma sobrelevação maior na busca pelo valor de referência. O controlador PI velocidade conseguiu manter e estabilizar o atraso na referência, mas apresentou muita perturbação na faixa em que o atraso do fluxo de voz tende a se manter muito abaixo da referência.

\section{PROPOSTA}

A proposta do presente artigo consiste em um novo paradigma no controle de QoS em redes IP, com o controle rígido do atraso das aplicações mais prioritárias através do controlador desenvolvido. O objetivo não é somente minimizar este atraso, mas estabelecer um valor máximo aceitável em caso de congestionamento da rede, que será configurado de acordo com as políticas estabelecidas.

\section{A. Definição do controlador proposto}

A partir dos resultados preliminares das simulações da Subseção IV.B, propõe-se um controlador que, nos instantes iniciais da busca ao valor de referência se comporte como um controlador do tipo $\mathrm{P}$ posicional e, ao atingir uma certa estabilidade passe a se comportar como um controlador do tipo PI velocidade. Vale ressaltar que na faixa em que o atraso da variável controlada se mantém muito abaixo do valor de referência, por características da planta, seria desejável que o controlador se comportasse como um controlador P posicional.

Para determinar a faixa de atuação dos controladores $\mathrm{P}$ e PI, utilizou-se a seguinte metodologia: quando o valor da diferenças entre os atrasos do fluxo de voz amostrados nos instantes $t$ e $t-1$ estiver na faixa de estabilização do sinal de $\pm 10 \%$ da referência $(\varphi)$ e atraso $>50 \%$ da referência (evitar faixa em que o atraso se mantém muito abaixo da referência - $\varphi$ ), de modo que o erro (atraso voz - referência) deverá ser maior que $-50 \%$ da referência, o controlador atuante será o controlador PI velocidade. Nos demais casos, o controlador atuante será o controlador $P$ posicional. Essas regras para a atuação do controlador híbrido proposto estão refletidas na Equação 4:

$$
\operatorname{peso}(t)=\left\{\begin{array}{l}
\operatorname{peso}(t-1)+0,45^{*} K_{c}[e(t)-e(t-1)]+\frac{K_{c} T_{s}}{(1 / 1,2) * P_{c}} e(t), \\
\operatorname{se}|\operatorname{atraso}(\mathrm{t})-\operatorname{atraso}(\mathrm{t}-1)|<10 \% . \varphi \mathrm{e} \mathrm{e}(\mathrm{t})>-50 \% . \varphi, \\
0,5 * K_{c} e(t)+\operatorname{peso}(0), \text { caso contrário. }
\end{array}\right.
$$

\section{B. Definição das políticas de controle}

Para efetuar testes de desempenho, foram estabelecidas políticas a serem aplicadas aos cenários analisados. O fluxo de voz foi definido como o fluxo de aplicação crítica, ou seja, o fluxo mais prioritário. $\mathrm{O}$ valor de referência para o atraso desejado para este fluxo deve ser previamente determinado dentro da faixa de atraso mínimo e máximo, conforme a Tabela II.

TABELA II - Parâmetros de QoS [6].

\begin{tabular}{|c|c|c|c|c|}
\hline \multicolumn{2}{|c}{ Parâmetro } & $\begin{array}{c}\text { Serviço } \\
\text { de Voz }\end{array}$ & $\begin{array}{c}\text { Serviço } \\
\text { de Dados }\end{array}$ & $\begin{array}{c}\text { Serviço de } \\
\text { Áudio e } \\
\text { Imagem }\end{array}$ \\
\hline $\begin{array}{c}\text { Itraso de } \\
\text { transmissão dos } \\
\text { pacotes }\end{array}$ & Min. & $100 \mathrm{~ms}$ & $1.000 \mathrm{~ms}$ & $100 \mathrm{~ms}$ \\
\cline { 2 - 5 } & Max. & $400 \mathrm{~ms}$ & $4.000 \mathrm{~ms}$ & $400 \mathrm{~ms}$ \\
\hline $\begin{array}{c}\text { Variação do } \\
\text { atraso dos } \\
\text { pacotes }\end{array}$ & Min. & $1 \mathrm{~ms}$ & $10 \mathrm{~ms}$ & $1 \mathrm{~ms}$ \\
\cline { 2 - 5 } & Max. & $100 \mathrm{~ms}$ & $100 \mathrm{~ms}$ & $100 \mathrm{~ms}$ \\
\hline \multirow{2}{*}{ Vazão } & Min. & $10 \mathrm{kbps}$ & $1 \mathrm{kbps}$ & $10 \mathrm{kbps}$ \\
\cline { 2 - 5 } & Max. & $64 \mathrm{k} \mathrm{bps}$ & $10 \mathrm{kbps}$ & $128 \mathrm{kbps}$ \\
\hline \multirow{2}{*}{$\begin{array}{c}\text { Taxa de perda } \\
\text { de pacotes }\end{array}$} & Min. & $10^{-4}$ & $10^{-6}$ & $10^{-4}$ \\
\cline { 2 - 5 } & Max. & $10^{-3}$ & $10^{-4}$ & $10^{-3}$ \\
\hline
\end{tabular}

A determinação do valor de referência do atraso escolhido para o fluxo de aplicação crítica (fluxo de voz) dependerá de decisões administrativas. Por exemplo, aplicações de telemedicina poderão ser agregadas nesta classe mais prioritária, na qual será estabelecido um controle rígido da QoS.

O fluxo de vídeo foi definido como o segundo fluxo mais prioritário. $\mathrm{O}$ valor de referência para o atraso deste fluxo foi relacionado com as características de descarte do fluxo menos prioritário (fluxo interferente) de acordo com as políticas estabelecidas para as simulações, conforme a Tabela III. 
TABELA III - Políticas dos valores de referência para o fluxo de vídeo.

\begin{tabular}{|c|c|}
\hline $\begin{array}{c}\text { Percentual descarte }(\mathrm{d}) \\
- \text { fluxo interferente }\end{array}$ & Atraso - fluxo de vídeo \\
\hline $\mathrm{d} \leq 10 \%$ & $100 \mathrm{~ms}$ \\
\hline $10 \%<\mathrm{d} \leq 20 \%$ & $150 \mathrm{~ms}$ \\
\hline $20 \%<\mathrm{d} \leq 30 \%$ & $200 \mathrm{~ms}$ \\
\hline $30 \%<\mathrm{d} \leq 40 \%$ & $250 \mathrm{~ms}$ \\
\hline $40 \%<\mathrm{d} \leq 50 \%$ & $300 \mathrm{~ms}$ \\
\hline $\mathrm{d}>50 \%$ & $350 \mathrm{~ms}$ \\
\hline
\end{tabular}

\section{RESUltados}

O desempenho do controlador proposto é analisado nas subseções a seguir (simulações $\alpha .1$ e $\alpha .2$ ), através da atuação nos fluxos de voz e vídeo conforme as políticas estabelecidas na Tabela III. O valor de referência escolhido para fluxo de voz foi de $200 \mathrm{~ms}$, o qual está condizente com a Tabela II - maior que o limiar mínimo (100 ms) e abaixo do limiar máximo (400 ms).

\section{A. Simulação $\alpha .1$}

A rede foi configurada com a taxa de tráfego interferente de 500 kbps e 6 fluxos do tipo FTP com transferência de arquivos com $3 \mathrm{MB}$ por fluxo, totalizando $18 \mathrm{MB}$. Os fluxos FTP foram estabelecidos pseudo-aleatoriamente com uma distribuição uniforme no intervalo de 45 a 50 segundos.

O peso do fluxo de FTP é igual a 20 e peso do fluxo interferente igual a 10. Para evitar problemas de sincronização, o controle do fluxo de voz inicia no instante de simulação igual a 2 segundos e o controle do fluxo de vídeo é disparado 8 segundos depois, i.e., em 10 segundos.

As taxas dos fluxos de voz e vídeo foram configuradas inicialmente com os valores $200 \mathrm{kbps}$ e 1 Mbps, respectivamente, e obedeceram à dinâmica descrita na Tabela IV.

TABELA IV - Dinâmica dos fluxos de voz e vídeo.

\begin{tabular}{|c|c|c|}
\hline Tempo de simulação & Taxa fluxo voz & Taxa fluxo vídeo \\
\hline $100 \mathrm{~s}$ & $200 \mathrm{kbps}$ & $900 \mathrm{kbps}$ \\
\hline $150 \mathrm{~s}$ & $200 \mathrm{kbps}$ & $1 \mathrm{Mbps}$ \\
\hline $200 \mathrm{~s}$ & $250 \mathrm{kbps}$ & $1 \mathrm{Mbps}$ \\
\hline $250 \mathrm{~s}$ & $200 \mathrm{kbps}$ & $1 \mathrm{Mbps}$ \\
\hline $300 \mathrm{~s}$ & $150 \mathrm{kbps}$ & $1 \mathrm{Mbps}$ \\
\hline
\end{tabular}

Observa-se na Figura 2 que o controlador mantém o atraso do fluxo de voz no valor de $200 \mathrm{~ms}$. No tempo de simulação $t=300 \mathrm{~s}$, o atraso do fluxo de voz se mantêm abaixo da referência estabelecida, devido a diminuição de sua vazão para $150 \mathrm{kbps}$ (vide Tabela IV) e a prioridade estabelecida tanto na arquitetura DiffServ quanto na ação do controlador. Os picos observados no atraso do fluxo de voz são devidos à ação do controlador respondendo às mudanças na dinâmica dos fluxos de voz e vídeo. Entretanto, estes picos estão dentro do limite máximo para fluxos de voz, conforme a Tabela II.

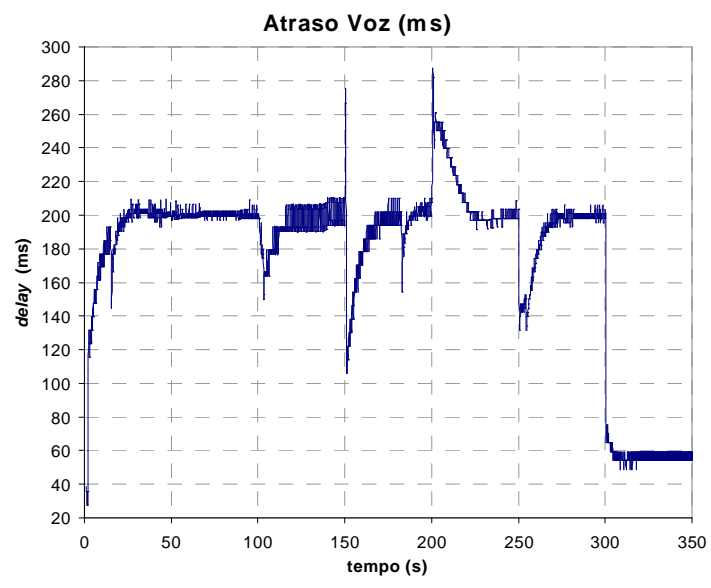

Fig. 2. Controlador voz/vídeo - atraso-voz.

$\mathrm{Na}$ Figura 3, pode-se observar o comportamento da variável de controle (peso voz) variando de forma a manter o valor do atraso de acordo com a referência estabelecida (200 ms). Quando o atraso do fluxo de voz tende a se manter abaixo da referência, a ação do controlador é de diminuir o valor do peso deste fluxo, de modo a transferir recursos da rede para os demais fluxos menos prioritários.

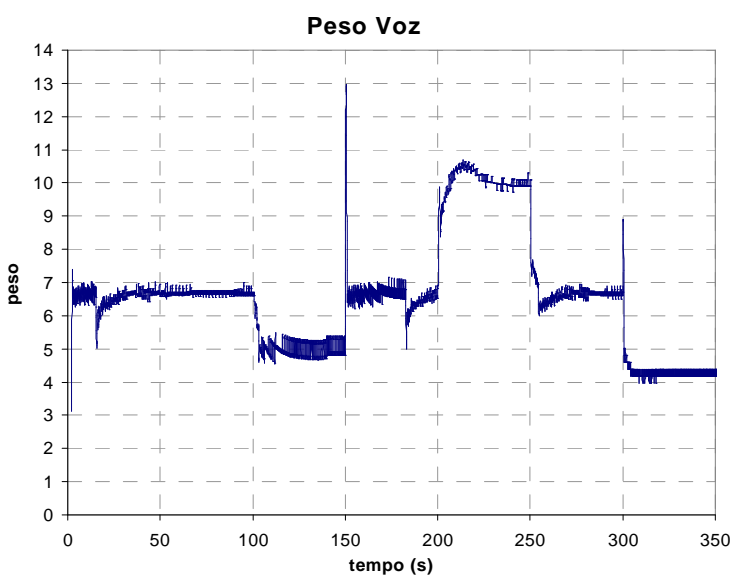

Fig. 3. Controlador voz/vídeo - peso-voz.

De acordo com a Figura 4, no instante $t=100 \mathrm{~s}$, quando a taxa do fluxo de vídeo diminui para $900 \mathrm{kbps}$ (vide Tabela IV), o valor do atraso do fluxo de vídeo se mantém abaixo da referência. O percentual de descarte do fluxo interferente se mantém entre $30 \%$ e $40 \%$ fazendo com que o valor de referência do atraso do fluxo de vídeo tenda a se manter na maior parte da simulação em $250 \mathrm{~ms}$, de acordo com as políticas definidas na Tabela III. 


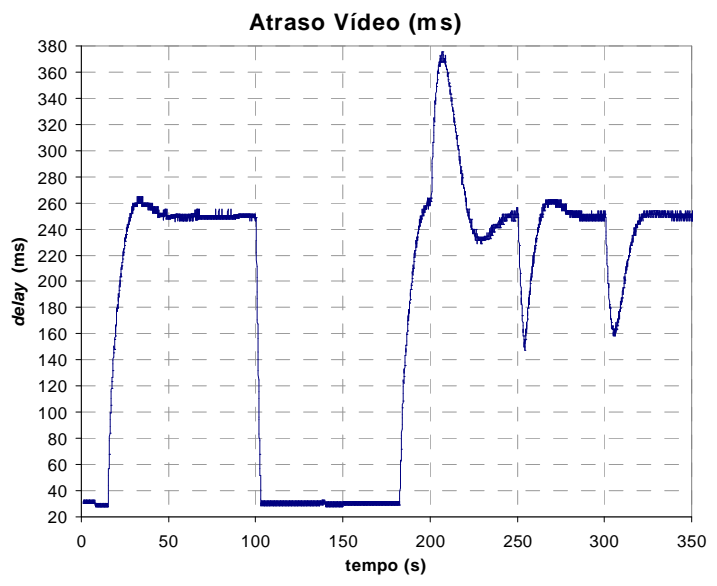

Fig. 4. Controlador voz/vídeo - atraso-vídeo.

Ocorre uma sobrelevação no atraso do fluxo de vídeo que chega a $370 \mathrm{~ms}$ (Figura 4), com uma duração longa, mais este valor está abaixo do limite máximo para fluxos de vídeo que é de $400 \mathrm{~ms}$, conforme a Tabela II. Com a diminuição do fluxo de voz nos tempos de simulação de $250 \mathrm{~s}$ e $300 \mathrm{~s}$, o atraso do fluxo de vídeo tende a diminuir e a ação do controlador é manter o atraso do fluxo de vídeo na referência estabelecida pelas políticas apresentadas na Tabela III. Como é necessário menos recurso da rede para manter o atraso do fluxo de vídeo na referência com a diminuição do fluxo de voz, a variável de controle pesovídeo é decrementada, liberando os recursos excedentes para os outros fluxos.

Na Figura 5 pode-se observar em $t=100 \mathrm{~s}$ a ação do controlador minimizando o valor da variável de controle peso-vídeo, transferindo os recursos excedentes para os demais fluxos. Em $t=200 \mathrm{~s}$, a taxa do fluxo de voz aumenta para $250 \mathrm{kbps}$ e o atraso do fluxo de vídeo tende a aumentar muito; então, para estabilizar o atraso do fluxo de vídeo, o valor da variável de controle peso-vídeo apresenta um rápido e acentuado incremento.

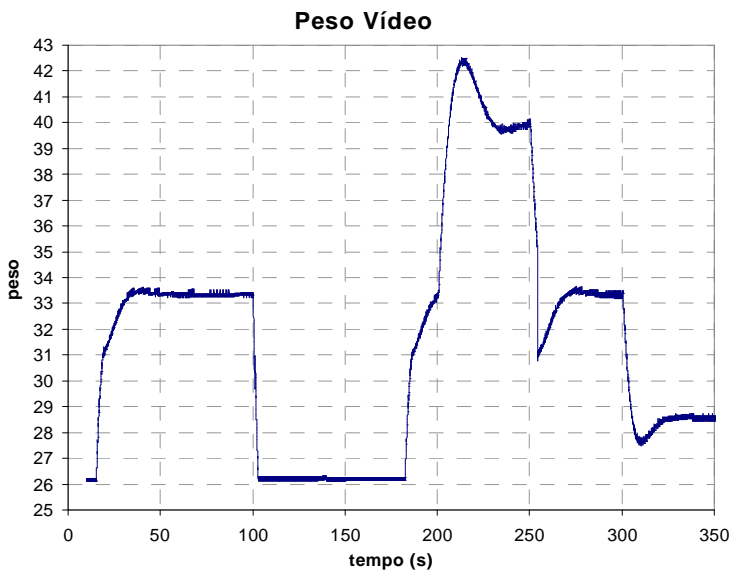

Fig. 5. Controlador voz/vídeo - peso-vídeo.

Nas Figuras 6 e 7 são mostradas as funções de distribuição cumulativa de probabilidade do jitter para os fluxos de voz e vídeo, respectivamente. Pode-se verificar a eficiência do controlador proposto em manter a variação do atraso dos fluxos de voz e vídeo em valores muito abaixo do limite máximo de $100 \mathrm{~ms}$, conforme a Tabela II. Em particular, para o fluxo de voz, praticamente $100 \%$ do jitter encontra-se abaixo de $10 \mathrm{~ms}$ (vide Figura 6). De modo semelhante, para o fluxo de vídeo, aproximadamente $100 \%$ do jitter encontra-se abaixo de 4 ms (vide Figura 7).

Os fluxos de voz e de vídeo não apresentam perda de pacotes e suas taxas de vazão não sofrem depreciação no enlace congestionado. Deste modo, o controlador proposto assegura largura de banda para estes fluxos. A taxa de perda de pacotes do fluxo interferente é de 35,91\%. A taxa de perda de pacotes referente ao enlace congestionado é de $10,67 \%$.

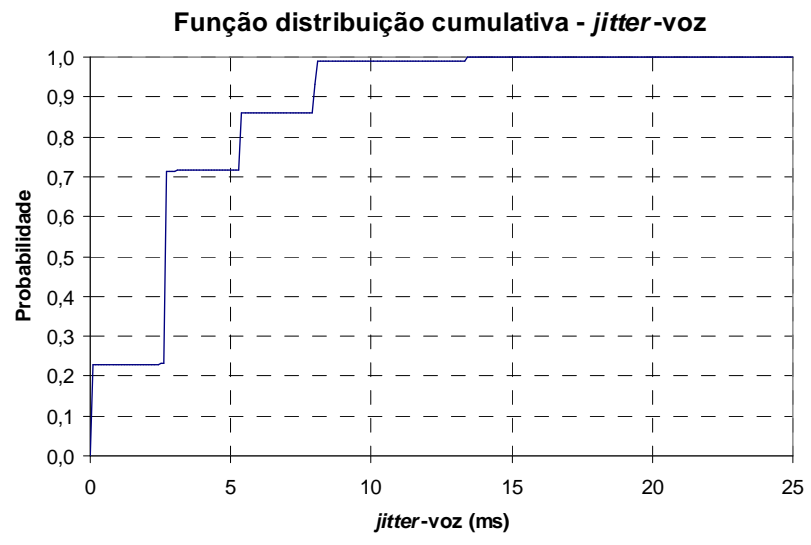

Fig. 6. Controlador voz/vídeo - jitter-voz.

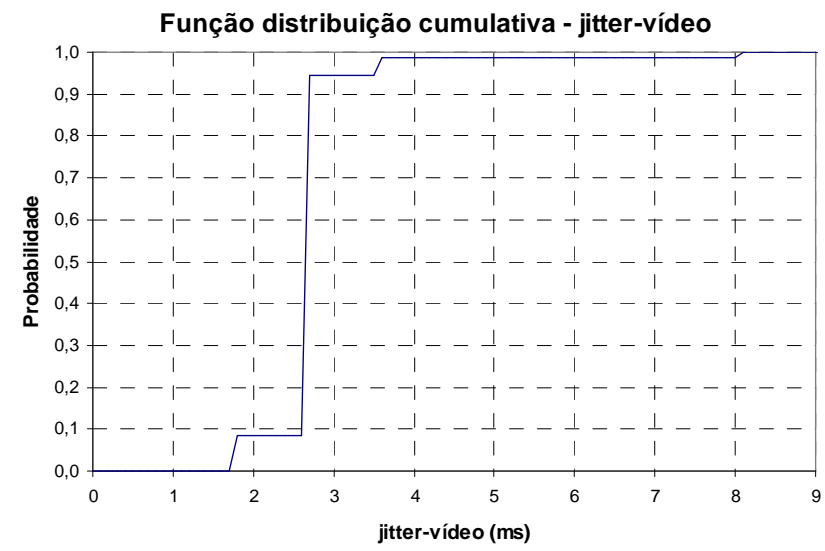

Fig. 7. Controlador voz/vídeo - jitter-vídeo.

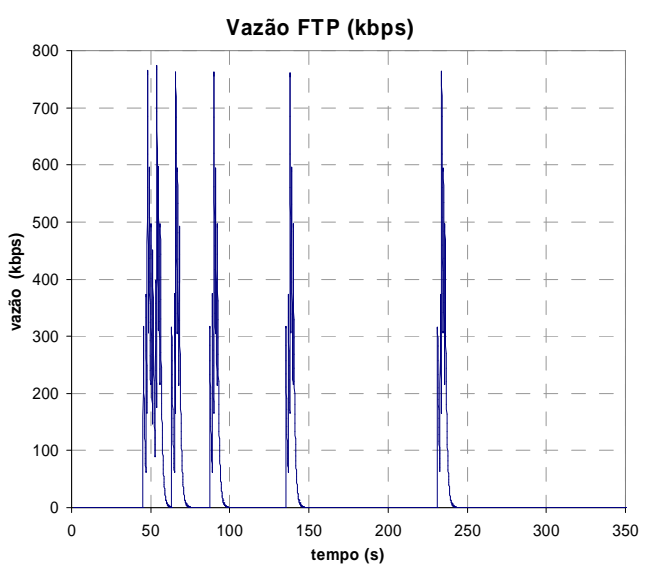

Fig. 8. Controlador voz/vídeo - vazão FTP.

Na Figura 8, observa-se que o fluxo FTP consegue atingir uma taxa de pico de vazão acima de $700 \mathrm{kbps}$. Como o fluxo FTP não apresentou perda de pacotes, não ocorreram 
retransmissões e as rajadas observadas indicam a transferência efetiva de dados pelo fluxo FTP.

\section{B. Simulação $\alpha .2$}

Essa simulação apresenta as mesmas configurações da simulação $\alpha .1$ (subseção precedente), sendo que a taxa do fluxo de voz se mantém em $200 \mathrm{kbps}$ e a taxa do fluxo de vídeo se mantém em $1 \mathrm{Mbps}$. O fluxo interferente inicia com uma taxa de $500 \mathrm{kbps}$, aumentando para $900 \mathrm{kbps}$ no instante de simulação igual a $200 \mathrm{~s}$.

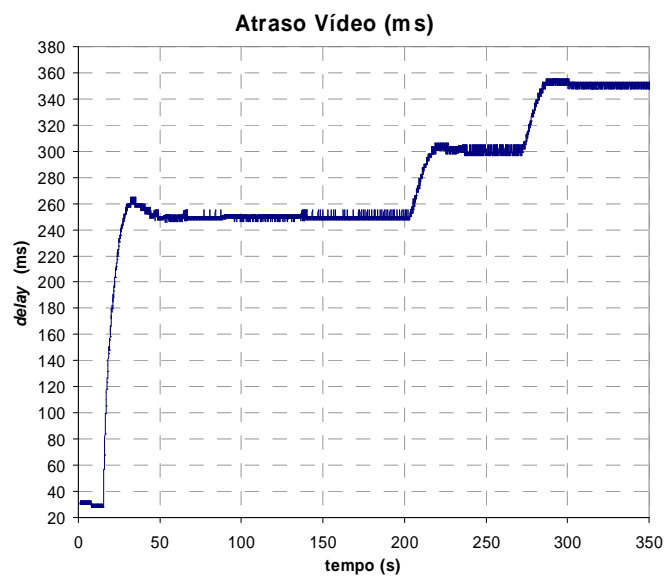

Fig. 9. Controlador voz/vídeo - atraso-vídeo ( $\alpha .2)$.

Nessa simulação, pode-se observar com maior clareza a influência das políticas adotadas relacionando os fluxos de vídeo e interferente. $\mathrm{O}$ valor de atraso do fluxo de vídeo de $250 \mathrm{~ms}$ está relacionado a uma taxa de descarte do fluxo interferente entre $20 \%$ e $30 \%$, conforme estabelecido na Tabela III. No instante $t=200 \mathrm{~s}$, a taxa do fluxo interferente aumenta para $900 \mathrm{kbps}$, fazendo ocorrer um aumento na taxa de descarte do fluxo interferente. Quando o valor da taxa de descarte do fluxo interferente passa para a faixa entre $30 \%$ e $40 \%$, o valor do atraso do fluxo de vídeo muda para $300 \mathrm{~ms}$. Com o aumento da taxa de descarte do fluxo interferente para valores acima de $40 \%$, o valor do atraso do fluxo de vídeo passa para $350 \mathrm{~ms}$. A dinâmica da variação do valor do atraso do fluxo de vídeo pode ser observada na Figura 9.

\section{CONCLUSÃO}

Neste trabalho, foi proposto um controlador clássico, nãolinear, para melhorar a provisão de QoS através de ações nas filas dos nós roteadores de um ambiente DiffServ bem definido. A simplicidade analítica da Equação 4, sua facilidade de implementação em código e sua baixa demanda de poder de processamento de máquina são pontos vantajosos. O controlador proposto apresentou resultados satisfatórios no controle dos parâmetros de QoS dos fluxos mais prioritários (atraso, jitter, perda de pacote, largura de banda).

Mediante o correto ajuste do peso da disciplina de enfileiramento WFQ, evitou-se a posse excessiva de recursos da rede pelos fluxos mais prioritários, melhorando a qualidade de serviço ofertada aos fluxos menos prioritários, diminuindo assim suas perdas de pacotes e melhorando suas métricas temporais (atraso e variação do atraso).

O uso de algoritmos adaptativos, e.g., controle gainscheduling ou técnicas de aprendizado de máquina (machine learning) podem ser considerados na perspectiva de se efetuar o ajuste dinâmico e a sintonia fina dos parâmetros dos controladores PID, diminuindo as sobrelevações da variável controlada (atraso). Pode-se até mesmo vislumbrálos como uma solução completa de gerência autonômica de uma rede IP. Em todo caso, pode-se afirmar que os resultados obtidos até aqui são bastante motivadores na medida em que apontam para a viabilidade do uso deste tipo de controladores para provimento de QoS de uma forma diferente e partitiva.

$\mathrm{Na}$ seqüência em curto prazo, pretende-se analisar o comportamento do controlador proposto com algumas das técnicas supracitadas, adicionando cenários mais complexos para averiguar o impacto devido ao fator escalabilidade.

\section{REFERÊNCIAS}

[1] Aguilar, Daniel Melchor; Torres, Víctor Castillo. "Stability Analysis of Proportional-Integral AQM controllers supporting TCP flows". Revista Computación $\quad$ y 2007. www.cic.ipn.mx/revistas/pages/vol10-04/v10no4_Art05.pdf

[2] Costa Filho, J. T.; Souza, C. P. "Controle por Computador Desenvolvendo Sistemas de Aquisição de Dados para PC'. $1^{\mathrm{a}}$ ed. São Luís: EDUFMA, 2001

[3] Drummond, André Costa. "Alocação de banda passante em redes auto-ajustáveis”. Dissertação de Mestrado, IC/Unicamp, 2005.

[4] Fernandez, Marcial Porto. "Provisionamento de Recursos em Arquitetura Diffserv para Melhoria da Qualidade de Serviço (QoS)". Tese de Doutorado. COPPE/UFRJ, D.Sc., Engenharia Elétrica, 2002.

[5] Gallardo, José R.; Makrakis, Dimitrios. "Dynamic Predictive Weighted Fair Queueing for Differentiated Services". Communications. 2001. ICC 2001. IEEE International Conference, 2001.

[6] Guido, Marcelo. "Propostas para Estender as Funcionalidades do RSVP-TE”. Instituto de Pesquisas Tecnológicas do Estado de São Paulo, 2004.

[7] Lima, Michele Mara de Araújo Espindula. "Projeto de Controladores Ótimos para Gerenciamento Ativo de Filas". Tese de Doutorado. Instituto de Computação Universidade Estadual de Campinas, 2005.

[8] Ogata, Katsuhiko. "Engenharia de Controle Moderno". Prentice Hall do Brasil. $4^{\text {a }}$ Edição. Rio de Janeiro - RJ, 2003.

[9] Panza, Gianmarco; Grazioli, Matteo; Sidoti, Filippo. "Design and analysis of a dynamic Weighted Fair Queuing (WFQ) scheduler". IST Mobile and Wireless Communication Summit '05, 2005.

[10] Panza, G.; Franco, C.; Lamy-Bergot, C.; Sidoti, F. "Weight updating methods for a dynamic Weighted Fair Queuing (WFQ) scheduler". IST Mobile and Wireless Communication Summit'06, 2006.

[11] Romão, Wesley; Niederauer, Carlos A. P.; Martins, Alejandro; Tcholakian, Aran; Pacheco, Roberto C. S. "Algoritmos Genéticos e Conjuntos Difusos Aplicados ao Controle de um Processo Térmico". Revista Tecnológica, n. 8, p. 7-21, 1999.

[12] “The Network Simulator - NS-2". http://www.isi.edu/nsnam/ns/.

[13] Wang, Lijun; Fayek, Dalia; Sivananthan, Thushyanth. "A Bandwidth Bargain Model based on Adaptive Weighted Fair Queueing". Network Qperations and Managemant Symposium. 2006. NOMS 2006. 10th IEEE/IFIP, 2006.

[14] Xu, Yue-Dong; Yang, Jie; Du, Qing. "Nonlinear PI active queue management based on hyperbolic secant functions". Machine Learning and Cvbernetics. Proceedinqs of 2005 International Conference on Volume 2, 18-21 Aug. 2005 Page(s):715 - 720 Vol. 2, 2005 . 